\title{
Pathology Associated with Adherent Perirenal Fat and its Clinical Effect
}

\author{
Erman Ceyhan ${ }^{1}$, Fatih Ileri ${ }^{1}$, Fazıl Tuncay Aki ${ }^{1}$, Mustafa Sertac Yazici ${ }^{1}$, Musturay \\ Karcaaltincaba $^{1}$, Dilek Ertoy Baydar ${ }^{2}$, and Cenk Yucel Bilen ${ }^{1}$ \\ ${ }^{1}$ Hacettepe University Faculty of Medicine \\ ${ }^{2}$ Koc Universitesi
}

March 3, 2021

\begin{abstract}
Introduction The dissection of perirenal fat is of critical importance to kidney surgery and ease of dissection is more important when using minimally invasive approaches. This study aimed to determine the clinical, radiological, and pathological significance of adherent perirenal fat(APF). Materials and Methods This prospective study included 22 patients scheduled for partial nephrectomy and 40 patients for donor nephrectomy. Intra-operative fat dissection time was recorded and the complexity of perirenal fat dissection was surgeon-classified as easy, moderate, and difficult. Perirenal fat and subcutaneous fat thickness was measured. Measurement of perirenal fat depth, and the Hounsfield unit(HU) for both perirenal and subcutaneous fields was performed using CT images. All specimens were submitted for histopatological analysis. Researchers in each arm were blinded to other researchers' data. Results Mean age of the patients was $51.3 \pm 12.7$ years. Mean perirenal fat dissection time was $15.0 \pm 13.5 \mathrm{~min}$. Patient demographics, BMI, nor occupational status differed between the 3 complexity of perirenal fat dissection groups. Radiological findings showed that there was a significant correlation between perirenal fat depth and complexity of perirenal fat dissection $(p<0.05)$, but not with HU measurements or subcutaneous fat thickness. Surgeon classification of the complexity of perirenal fat dissection was in accordance with the duration of dissection $(\mathrm{p}<0.05)$. Perinephric fat contained more fibrous tissue in the patients with histologically proven APF than in those without $(\mathrm{p}<0.05)$. Conclusions APF is a challenge during kidney surgery. Difficult dissection prolongs the duration of surgery, which can lead to complications. Perirenal fat thickness measured via pre-operative CT might be used to predict APF.
\end{abstract}

Pathology Associated with Adherent Perirenal Fat and its Clinical Effect

Erman CEYHAN 1o, Fatih ILERI ${ }^{1}$, Fazil Tuncay AKI $^{1}$, Mustafa Sertac YAZICI ${ }^{1}$, Musturay KARCAALTINCABA $^{2}$, Dilek ERTOY BAYDAR ${ }^{3}$, Cenk Yucel BILEN ${ }^{1}$

${ }^{1}$ Hacettepe University, School of Medicine, Department of Urology, Ankara, Turkey

${ }^{2}$ Hacettepe University, School of Medicine, Department of Radiology, Ankara, Turkey

${ }^{3}$ Koç University, School of Medicine, Department of Pathology, Istanbul, Turkey

Running title: Adherent Perirenal Fat

Declarations of interest: None.

Acknowledgments: This study did not received any funding. Authors have nothing to declare.

Manuscript word count: 2985

Abstract word count: 250 
Author contributions:

Research conception and design: EC, FI, MK, DEB, . Data acquisition: EC, FI, FTA, MSY, MK, DEB, . Statistical analysis: EC, FI, DEB,. Data analysis and interpretation: EC, DEB, . Drafting of the manuscript: EC, DEB, CYB. Critical revision of the manuscript: EC, FTA, MSY, DEB, CYB. Obtaining funding: N/A. Administrative, technical, or material support: EC,. Supervision: DEB, CYB. Approval of the final manuscript: EC, FI, FTA, MSY, DEB, CYB .

${ }^{\circ}$ Corresponding author: Asst. Prof. Erman Ceyhan M.D., FEBU

Mailing address: Baskent University Konya Hospital, Department of Urology

Hocacihan Mah. Saray Cad. No: 1, 42080 Selçuklu, Konya / Turkey

Tel: $00903322570600 / 3532-3536$

Fax: 00903322570637

Mobile phone: 00905355873688

E-mail:erman_ceyhan@yahoo.com

Pathology Associated with Adherent Perirenal Fat and its Clinical Effect

\section{Abstract}

\section{Introduction}

The dissection of perirenal fat is of critical importance to kidney surgery and ease of dissection is more important when using minimally invasive approaches. This study aimed to determine the clinical, radiological, and pathological significance of adherent perirenal fat(APF).

\section{Materials and Methods}

This prospective study included 22 patients scheduled for partial nephrectomy and 40 patients for donor nephrectomy. Intra-operative fat dissection time was recorded and the complexity of perirenal fat dissection was surgeon-classified as easy, moderate, and difficult. Perirenal fat and subcutaneous fat thickness was measured. Measurement of perirenal fat depth, and the Hounsfield unit(HU) for both perirenal and subcutaneous fields was performed using CT images. All specimens were submitted for histopatological analysis. Researchers in each arm were blinded to other researchers' data.

\section{Results}

Mean age of the patients was $51.3 \pm 12.7$ years. Mean perirenal fat dissection time was $15.0 \pm 13.5 \mathrm{~min}$. Patient demographics, BMI, nor occupational status differed between the 3 complexity of perirenal fat dissection groups. Radiological findings showed that there was a significant correlation between perirenal fat depth and complexity of perirenal fat dissection $(\mathrm{p}<0.05)$, but not with HU measurements or subcutaneous fat thickness. Surgeon classification of the complexity of perirenal fat dissection was in accordance with the duration of dissection $(\mathrm{p}<0.05)$. Perinephric fat contained more fibrous tissue in the patients with histologically proven APF than in those without $(\mathrm{p}<0.05)$.

\section{Conclusions}

APF is a challenge during kidney surgery. Difficult dissection prolongs the duration of surgery, which can lead to complications. Perirenal fat thickness measured via pre-operative CT might be used to predict APF.

Keywords : Perirenal fat; dissection; nephrectomy; minimally invasive surgical procedures; kidney

What is already known about this topic?

The ways of prediction of adherent perirenal fat before surgery have been studied previously with complex scoring systems but there is only one study which adresses the histopathology under this phenomenon. 
What does this article add?

Results of this study revealed that there is no need for complex scoring systems in order to predict adherent perirenal fat. Perirenal fat thickness measured via pre-operative CT can predict adherent perirenal fat single handedly. Also detailed histopathological investigation under adherent perirenal fat has been reported by this study.

\section{Abbreviations:}

APF: Adherent perirenal fat

CT: Computed tomography

BMI: Body mass index

HU: Hounsfield unit

MAP: Mayo Adhesive Probability

\section{Introduction}

The dissection of perirenal fat is of critical importance to kidney surgery, especially in cases of partial and donor nephrectomy. Good dissection of perirenal fat provides good exposure, easy manipulation, and shorter surgical duration ${ }^{1,2}$. In $10.6 \%-55.2 \%$ of cases perirenal fat tissue is adhered to the kidney, which makes it difficult to dissect; this phenomenon is referred to as adherent perirenal fat(APF) or sticky perirenal fat ${ }^{2}$.

Patient-related factors, including gender, and visceral and perinephric fat thickness are clinically associated with the complexity of surgery and surgical outcome ${ }^{1,3}$. For predicting the complexity of perirenal fat dissection many researchers suggest using such tools as renal scoring systems and pre-operative computed tomography (CT) measurement ${ }^{4,5}$. Prediction of APF can help in choosing the optimal surgical approach. Severe APF can complicate and hinder minimally invasive surgery.

All relevant studies in the literature have emphasized the prediction of APF pre-operatively, with very little attention to the underlying pathophysiology. Recently, Dariane et al. published a study on the histological changes linked to $\mathrm{APF}^{6}$. The present study aimed to determine the clinical, radiological, and pathological significance of APF.

\section{Materials and Methods}

\section{Patient selection}

This prospective study included patients that were scheduled for partial nephrectomy or donor nephrectomy between October 2016-June 2017. Patients with a history of renal interventions and recurrent urinary tract infection, and those without CT images were excluded from the study. After exclusion of 6 patients, the remaining 62 adults were included, of which 22 underwent partial nephrectomy and 40 underwent donor nephrectomy. Type of surgical approach was chosen according to surgeons preference and experience. The study was carried out with 3 arms; clinical phase; radiological measurement; histopathological examination. The researchers in each arm were blinded to each other's data. The study protocol was approved by our institutional ethics committee (IRB No:GO16/669-26). All the patients provided written informed consent before undergoing surgical intervention.

\section{Data collection}

Clinical data collection and intra-operative measurements were performed by the same researcher(EC). Patient demographics, weight, height, body mass index(BMI), and occupational status were recorded. Occupational status was categorized as light workers and heavy workers. Patients that worked in jobs requiring physical strength, such as construction, farming, delivery, were categorized as heavy workers, whereas office employees, housewives, and the unemployed were categorized as light workers. 
Surgery was performed by experienced surgeons (FTA, CYB or MSY). All nephrectomies were performed via the transperitoneal approach. In order to record surgical data the researcher in charge (EC) and an observer(FI) were present during all surgical procedures. Perirenal fat dissection time was recorded by a time observer (FI). The complexity of perirenal fat dissection was classified by EC and divided into 3 categories; easy, moderate, and difficult. This classification was based on the difficulty of dissection, capsular trauma during dissection, bleeding, and unintentional injuries during removal of perirenal fat from the kidney. In addition, maximum perirenal fat thickness $(\mathrm{mm})$ and maximum subcutaneous fat thickness $(\mathrm{mm})$ were measured intra-operatively by EC. The removed perirenal fat tissue was sent for histopathological examination after measurement.

\section{Radiology}

All radiological measurements were performed by the same radiologist(MK). Perirenal fat thickness was measured at the level of the renal vein, from the posterior renal capsule to the posterior abdominal wall using CT images. These measurements from CT images are referred to as perirenal fat depth to eliminate confusion. The perirenal field Hounsfield unit(HU) and subcutaneous fat HU(at the level of the hilus) were recorded according to a circular region of interest measuring $1 \mathrm{~cm}$ in diameter, using a PACS workstation and pre-operative $\mathrm{CT}$ images.

\section{Pathology}

Following overnight fixation in $4 \%$ formaldehyde at room temperature, resected fat tissue was subjected to a routine paraffin embedding procedure. Sections $5 \mu \mathrm{m}$ thick were obtained from paraffin blocks and initially stained with hematoxylin-eosin using a Shandon Varistain Gemini@(Shandon, Frankfurt) automated slide stainer. Additional histochemical staining with periodic acid methenamine silver and Masson's trichrome was performed to assess morphology and the degree of fibrosis in greater detail. All light microscopic evaluations were conducted using an Olympus BX53ß) optical microscope. Measurements were performed using digital photos captured using an Olympus SC50 CMOSß camera and the drawing line tools in CellSens Entry Imaging v.1.13®(Olympus Europa SE \& Co. KG, Hamburg). Adipocyte diameter was manually drawn and individually measured. In total, 350 adjacent adipocytes were measured in each patient. Cells $<35 \mu$ m were excluded from analysis to avoid immature multilocular adipocytes. Maximum adipocyte diameter and the presence of brown fat were noted separately.

During microscopic examination the presence of fibrosis was observed in some cases, primarily in the septae between fat lobules(i.e., perilobular fibrosis), around blood vessels(i.e., perivascular fibrosis), and/or in the outermost Gerota's fascia(Figure 1). Collagen fibers were organized in bundles of variable thickness and some exhibited spiculated infiltration to the periphery of the fat lobules, isolating a few adipocytes from the remainder of the tissue. Moreover, some cases exhibited pericellular fibrosis consisting of thinner collagen fibrils surrounding adipocytes, localized in areas far from fibrotic bundles(Figure 2). The presence or absence of these features was recorded in each case. Additionally, maximum thickness of the collagen at Gerota's fascia and wall thickness of the thickest vessel(from the endothelia to adventitia) in the resected fat were measured, as described above. The number of vessels with a thick wall $(>100 \mu \mathrm{m})$ in a 1-cm² area varied according to patient and was scored as follows: 1: $<3$ vessels; 2: 3-5 vessels; 3: [?]6 vessels . Minimal inflammatory infiltrates that contained a small number of lymphocytes and/or histiocytes existed in a few cases and was also recorded.

Immunohistochemical staining with primary anti-CD31 antibody(Leica Biosystems@); dilution,1:100; product code: NCL-L-CK7-560), an endothelial marker, was performed to evaluate microvessel density in perinephric fat. The Leica BOND-Max@ automated staining platform (Leica Microsystems, Wetzlar, Germany) and Bond Polymer Refine Detection Kit@(Leica Biosystems, Newcastle Ltd., Newcastle Upon Tyne, UK; cat. no: DS9800) were used for this purpose. In each case, 3 fields with the highest number of microvessels were identified at low power. In these areas mean capillary density (ACD) at $400 \times$ magnification was determined and expressed as the number of CD31-positive capillaries per high power field.

Specimens were qualified for histopathological examination in $55(88.7 \%)$ of the 62 cases; 7 samples were 
excluded from the study due to poor sampling or technical problems.

\section{Statistical analysis}

Statistical analysis was performed using IBM SPSS Statistics for Windows v.23®) (IBM Corp., Armonk, N.Y., USA). Continuous variables are shown as mean \pm SD and median(range). One-way ANOVA was used to analyze parametric variables, versus the Kruskal-Wallis test for non-parametric quantitative variables. Comparison of categorical variables was performed using the Chi-square test. Correlation between nonparametric data was analyzed by Spearman' correlation analysis. The level of statistical significance was set at $\mathrm{p}<0.05$.

\section{Results}

Mean age of the patients was $51.3 \pm 12.7$ years and the male to female ratio was $34: 28$. Mean BMI was $28.6 \pm 5.2$; among the 62 patients, 23 were overweight and 26 were obese. In total, $3 / 62$ patients had diabetes mellitus, $15 / 62$ had hypertension, and 2/62 were smokers. Among the patients, $90.3 \%(\mathrm{n}=56 / 62)$ were light workers. Among the 22 partial nephrectomies, $17(77.3 \%)$ were performed via an open approach and $5(22.7 \%)$ were performed laparoscopically. All 40 donor nephrectomies were performed laparoscopically, of which 36/40(90\%) were hand assisted. Most of the nephrectomies were performed on the left side(52/62). Mean tumor diameter was $35.8 \pm 16.7 \mathrm{~mm}(\mathrm{n}=22)$.

The distribution of cases according to dissection complexity was as follows: easy: $\mathrm{n}=27(43.5 \%)$; moderate: $\mathrm{n}=20(32.3 \%)$; difficult: $\mathrm{n}=15(24.2 \%)$. Mean perirenal fat dissection time was $15.0 \pm 13.5$ min. Mean dissection time in the cases with easy, moderate, and difficult dissection complexity was $5.6 \pm 2.9,12.7 \pm 2.1$, and $35.1 \pm 13.0 \mathrm{~min}$ respectively. Dissection complexity classification(as easy, moderate and hard) was in correlation with the time required for the dissection $(\mathrm{p}<0.05)$.

There wasn't a significant difference in perirenal fat dissection complexity between the male and female patients $(p=0.074)$. Similarly, correlations between weight, height, and BMI were not significant. Moreover, there wasn't a correlation between perirenal fat dissection complexity, and surgical side, the presence of diabetes mellitus, the presence of hypertension, smoking status, or occupational status $(\mathrm{p}>0.05)$.

The was a significant difference in perirenal fat dissection complexity between open and laparoscopic nephrectomies $(\mathrm{p}<0.05)$; most of the difficult dissection complexity cases underwent open surgery. Subcutaneous fat thickness measured intra-operatively did not differ according to dissection complexity $(\mathrm{p}=0.87)$; however, intra-operative measurement of perirenal fat thickness and dissection complexity were significantly correlated, such that median perirenal fat thickness was lowest in the cases with easy dissection complexity(median: 10,4-20) group and highest in those with difficult dissection complexity(median: 17, 10-28, $\mathrm{p}<0.05$ ).

Radiological findings showed that there was a significant relation between perirenal fat depth and perirenal fat dissection complexity; thewith a higher difference was greatest in the difficult dissection complexity patients $(\mathrm{p}<0.05)$; however, HU for the perirenal field and subcutaneous fat measured via CT showed that there wasn't a significant difference according to dissection complexity $(\mathrm{p}=0.88$ and $\mathrm{p}=0.52$, respectively). The radiologically measured perirenal fat depth and intra-operative measurement of perirenal fat thickness were significantly correlated $(\mathrm{r}=0.363, \mathrm{p}=0.005)$.

Perinephric fat exhibited evidence of histological fibrosis more commonly in the patients with severe APF(Table). All 4 parameters that we explored for fibrosis were higher in the patients with difficult dissection

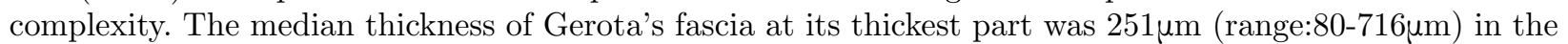

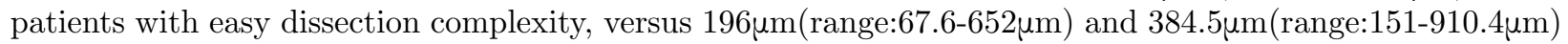

in those with moderate and difficult dissection complexity, respectively( $\mathrm{p}=0.006)$. In all, $8(34.8 \%)$ of the 23 patients with easy dissection complexity, 5(29.4\%) of the 17 with moderate dissection complexity, and $12(80 \%)$ of the 15 with difficult dissection complexity had perivascular fibrosis $(\mathrm{p}=0.007)$. Intercellular collagen accumulation was observed in $8.7 \%(2 / 23)$ of the patients with easy dissection complexity, $11.8 \%(2 / 17)$ of those with moderate dissection complexity, and $80 \%(12 / 15)$ of those with difficult dissection complex$\operatorname{ity}(\mathrm{p}=0.000)$. 
Spicules or bands of collagen radiating from the outer fascia, interlobular septae, and perivascular fibrous tissue were more common in the patients with $\operatorname{APF}(22 \%, 47 \%, 87 \%$ in the easy, moderate, and difficult dissection complexity patients, respectively, $\mathrm{p}=0.000$ ). There weren't any significant differences in inflammation, or mean and maximum adipocyte diameter according to perirenal fat dissection complexity. Fat tissue in the severe APF patients contained a higher number of blood vessels with wall thickness $>100 \mu \mathrm{m}(\mathrm{p}=0.039)$. There wasn't a significant difference in small capillary density according to perirenal fat dissection complexity $(\mathrm{p}>0.05)$.

\section{Discussion}

APF is a clinical phenomenon without a direct effect on patients, yet for surgeons it complicates dissection and release of the kidney ${ }^{7,8}$. Dissection of perirenal fat is an important component of partial nephrectomy and donor nephrectomy. APF can cause excessive hemorrhaging during surgery, trauma to the renal capsule, iatrogenic vascular injury, and prolongation of operative time $e^{6,8,9}$. Hemorrhaging from fatty tissue and vascular injury can complicate endourological manipulations. During laparoscopic and robot-assisted partial nephrectomy APF impedes kidney dissection. Furthermore, prolonged surgery time and renal capsular trauma can interfere with the renal transplantation process. Thusly, APF is a challenging phenomenon, both for open and endoscopic procedures. In addition to the radiological features, the present study focused on the pathological aspects of APF.

In the most recent studies APF was qualified as present or absent; only Narita et al. classified cases as non$\mathrm{APF}, \mathrm{APF}$, and severe APF, as in the present study ${ }^{10-12}$. We acknowledge that APF differs in severity from patient to patient. Dissection in some patients is sometimes so difficult that decapsulation occurs multiple times and occasionally complete removal of perirenal fat cannot be achieved; however, in some cases with APF dissection is managed with only minimal trauma and hemorrhaging. For the aforementioned reasons we find that it is appropriate to characterize APF as easy, moderate, and difficult.

The correlation between perirenal fat dissection complexity and dissection time in the present study confirms the pertinence of the dissection classification used. Zheng et al. classified APF according to dissection duration, as $<10 \mathrm{~min}, 10-20 \mathrm{~min}$, and $>20 \mathrm{~min}^{13}$. Their dissection time groupings were comparable to those in the present study, in which mean dissection time in the easy, moderate, difficult dissection complexity patients was $5.6 \pm 2.9 \mathrm{~min}, 12.7 \pm 2.1 \mathrm{~min}$, and $35.1 \pm 13.0 \mathrm{~min}$, respectively.

Retroperitoneal fat thickness is reported to affect duration of surgery, as it did in the present study. In addition to male gender, Ito et al. showed that retroperitoneal fat thickness is positively correlated with surgical duration $^{3}$. In a prospective study of 100 robotic partial nephrectomies it was reported that surgery time in patients with $\mathrm{APF}$ was longer than in those without $\mathrm{APF}^{12}$. A study that included 92 donor nephrectomies observed that APF and severe APF significantly prolonged surgery ${ }^{10}$. In the present study dissection time was significantly affected by APF; dissection time was longest in the patients with difficult dissection complexity. Total duration of surgery was not a focus of the present study because it can be deceptive. Total surgery time in donors during the transplantation process is affected by many factors; as such, we think that dissection time is a more reliable measurement for assessment of APF.

For the prediction of APF the image-based Mayo Adhesive Probability(MAP) score has been introduced ${ }^{4}$. This 5-point scoring system uses posterior perinephric fat thickness and perinephric fat stranding. The utility of the MAP score has been verified by several researchers ${ }^{7,14}$. Furthermore, many studies show that there is a clear association between APF and perirenal fat thickness ${ }^{6,10,11}$. Perirenal stranding observed in radiological images is not always an indicator of APF and stranding is a subjective parameter. To assess the clinical relevance of the perirenal field's radiological features, the perirenal field's HU was measured in the present study, and there wasn't a significant correlation between the perirenal field's HU and APF, yet perirenal fat depth differed significantly according to the complexity of perirenal fat dissection. Based on the present findings, we think use of perirenal fat depth measured via pre-operative CT is sufficient for presurgical prediction of APF.

Obesity is thought to play a role in APF, yet there wasn't a significant correlation between APF and BMI 
in the present study. Correspondingly, 2 other studies did not note an association between BMI and APF; however, others have observed a marked association ${ }^{6,8,11,14,15}$. Some studies considered waist circumference a predictor of $\mathrm{APF}^{6}$. The present study analyzed subcutaneous fat thickness as an indicator of internal fat burden, which is assumed to be a more objective parameter than waist circumference, but there wasn't a significant association between subcutaneous fat thickness and APF.

In addition to a high BMI, many other factors are associated with APF. Khene et al. assessed APF in 202 patients that underwent robot-assisted partial nephrectomy and reported that male gender, old age, a high $\mathrm{BMI}$, smoking, and hypertension are risk factors for $\mathrm{APF}^{8}$. Similarly, Kawamura et al. reported the same risk factors for $\mathrm{APF}^{14}$. Kocher et al. noted a significant association between $\mathrm{APF}$, and increased age and male gender ${ }^{11}$. In the present study there wasn't a correlation between APF and any of the aforementioned factors. The donor population in the present study might account for these differences, as the renal donors were for the most part fit and healthy non-smokers.

Radiologically measured posterior fat thickness is a predictor of $\mathrm{APF}^{10,11}$. Additionally, a prospective study that included 125 patients reported that lateral fat thickness was greater in patients with $\mathrm{APF}^{6}$. Moreover, increased posterior fat thickness, visceral fat area, and perinephric fat area are reported to be risk factors for $\mathrm{APF}^{10,15}$. In the present study perirenal fat thickness and perirenal fat depth measured via CT differed significantly according to perirenal fat dissection complexity. In the present study measurement of actual perirenal fat thickness was performed in order to detect the thickest part of the fatty tissue and perirenal fat depth was measured in order to use a more standardized measure. HU measurements of the perirenal field and subcutaneous fat via CT in the present study showed no significant difference according to dissection complexity. Histopathological findings supported this finding, no difference was observed in small capillary density according to dissection complexity. Bylund et al. reported a similar finding; however, Dariane et al. indicated the density of fat was an independent predictor of $\mathrm{APF}^{6,15}$.

Histopathlogical analysis in the present study show increased fibrous tissue in the perirenal fat of APF patients, in contrast to the findings reported by Dariane et $\mathrm{al}^{6}$. This seems to be the major underlying factor that can lead to surgical difficulty. Thicker Gerota's fascia, and more collagen around blood vessels and in between fat cells focally were observed in the present study's severe APF patients, but what caused this variance is not clear. Metabolic disorders, such as obesity and diabetes, and collagen vascular diseases or other systemic problems might have led to an increase in collagen deposition in the tissues; however, the present study's APF and non-APF patients did not differ in these respects. Additionally, there wasn't a significant difference in mean fat cell diameter, indicating that obese or overweight patients have not accumulated on one arm of the study set. Furthermore, we could not attribute to an inflammatory process for the explanation of fibrosis as both groups of patients have only a very few mononuclear cells over and there. In fact, we think that the amount of fibrous tissue might have been innate and related to the individual's features. The present finding that the APF patients had a higher number of blood vessels with wall thickness $>100 \mu \mathrm{m}$ might be considered supportive of this hypothesis.

The primary limitation of the present prospective study is the small number of patients. As living donor nephrectomy is performed less frequently, the study also included partial nephrectomies. Pathological examination might have been more objective if it had been conducted in donors only.

\section{Conclusions}

APF is a challenge for kidney surgeons. Difficult perirenal fat dissection prolongs surgical duration and can lead to complications. Predicting APF pre-operatively helps in choosing which patients qualify for minimally invasive surgery. Perirenal fat thickness measured via pre-operative CT can predict APF. There is a positive correlation between perirenal fat thickness and perirenal fat dissection complexity.

Acknowledgments: This study did not received any funding. Authors have nothing to declare.

Disclosure of interest: The authors report no conflicts of interest.

\section{REFERENCES}


1. Macleod LC, Hsi RS, Gore JL, Wright JL, Harper JD. Perinephric fat thickness is an independent predictor of operative complexity during robot-assisted partial nephrectomy. Journal of endourology.2014;28(5):587591.

2. Lee S-M, Robertson I, Stonier T, Simson N, Amer T, Aboumarzouk OM. Contemporary outcomes and prediction of adherent perinephric fat at partial nephrectomy: a systematic review. Scand J Urol.2017;51(6):429434.

3. Ito H, Makiyama K, Kawahara T, et al. The impact of gender difference on operative time in laparoscopic partial nephrectomy for T1 renal tumor and the utility of retroperitoneal fat thickness as a predictor of operative time. BMC Cancer. 2016;16(1):944.

4. Davidiuk AJ, Parker AS, Thomas CS, et al. Mayo adhesive probability score: an accurate image-based scoring system to predict adherent perinephric fat in partial nephrectomy. Eur Urol.2014;66(6):1165-1171.

5. Martin L, Rouviere O, Bezza R, et al. Mayo adhesive probability score is an independent computed tomography scan predictor of adherent perinephric fat in open partial nephrectomy. Urology.2017;103:124128.

6. Dariane C, Le Guilchet T, Hurel S, et al. Prospective assessment and histological analysis of adherent perinephric fat in partial nephrectomies. Paper presented at: Urologic Oncology: Seminars and Original Investigations2017.

7. Ishiyama R, Kondo T, Takagi T, et al. Impact of the Mayo adhesive probability score on the complexity of robot-assisted partial nephrectomy. J Endourol. 2018;32(10):928-933.

8. Khene Z-E, Peyronnet B, Mathieu R, Fardoun T, Verhoest G, Bensalah K. Analysis of the impact of adherent perirenal fat on peri-operative outcomes of robotic partial nephrectomy. World J Urol.2015;33(11):18011806.

9. Gorin MA, Mullins JK, Pierorazio PM, Jayram G, Allaf ME. Increased intra-abdominal fat predicts perioperative complications following minimally invasive partial nephrectomy. Urology.2013;81(6):1225-1231.

10. Narita S, Kumazawa T, Tsuchiya N, et al. Host-related risk factors for adherent perinephric fat in healthy individuals undergoing laparoscopic living-donor nephrectomy. Surg Laparosc Endosc Percutan Tech. 2017;27(4):e69.

11. Kocher NJ, Kunchala S, Reynolds C, Lehman E, Nie S, Raman JD. Adherent perinephric fat at minimally invasive partial nephrectomy is associated with adverse peri-operative outcomes and malignant renal histology. BJU international. 2016;117(4):636-641.

12. Davidiuk AJ, Parker AS, Thomas CS, Heckman MG, Custer K, Thiel DD. Prospective evaluation of the association of adherent perinephric fat with perioperative outcomes of robotic-assisted partial nephrectomy. Urology. 2015;85(4):836-842.

13. Zheng Y, Espiritu P, Hakky T, Jutras K, Spiess PE. Predicting ease of perinephric fat dissection at time of open partial nephrectomy using preoperative fat density characteristics. BJU international.2014;114(6):872880.

14. Kawamura N, Saito K, Inoue M, et al. Adherent perinephric fat in Asian patients: Predictors and impact on perioperative outcomes of partial nephrectomy. Urol Int. 2018;101(4):437-442.

15. Bylund JR, Qiong H, Crispen PL, Venkatesh R, Strup SE. Association of clinical and radiographic features with perinephric "sticky" fat.J Endourol. 2013;27(3):370-373.

Table. Analysis of histological findings. 


\begin{tabular}{|c|c|c|c|c|}
\hline Variables & $\begin{array}{l}\text { Easy Dissection } \\
\text { Complexity } \\
\text { Group }(\mathrm{n}=23)\end{array}$ & $\begin{array}{l}\text { Moderate } \\
\text { Dissection } \\
\text { Complexity } \\
\text { Group }(\mathrm{n}=17)\end{array}$ & $\begin{array}{l}\text { Difficult } \\
\text { Dissection } \\
\text { Complexity } \\
\text { Group }(\mathrm{n}=15)\end{array}$ & $\mathrm{p}$ value \\
\hline $\begin{array}{l}\text { Presence of } \\
\text { inflammation, } \mathrm{n} \\
(\%)\end{array}$ & $2(8.7 \%)$ & $3(17.6 \%)$ & $4(26.7 \%)$ & 0.344 \\
\hline $\begin{array}{l}\text { Presence of } \\
\text { perivascular } \\
\text { fibrosis, n (\%) }\end{array}$ & $8(34.8 \%)$ & $5(29.4 \%)$ & $12(80 \%)$ & 0.007 \\
\hline $\begin{array}{l}\text { Capillary density } \\
\text { (per unit area) }\end{array}$ & $20(10-40)$ & $17(12-33)$ & $15(10-50)$ & 0.641 \\
\hline $\begin{array}{l}\text { Presence of brown } \\
\text { fat, } \mathrm{n}(\%)\end{array}$ & $6(26.1 \%)$ & $6(35.3 \%)$ & $1(6.7 \%)$ & 0.158 \\
\hline $\begin{array}{l}\text { The average } \\
\text { adipocyte } \\
\text { diameter, ?m } \\
\text { (range) }\end{array}$ & $101(56.5-150)$ & $84.8(62-153)$ & $109(72-203)$ & 0.130 \\
\hline $\begin{array}{l}\text { Maximum } \\
\text { adipocyte } \\
\text { diameter, ?m } \\
(\text { mean } \pm \mathrm{SD})\end{array}$ & $146.4 \pm 31.5$ & $139.1 \pm 30.2$ & $154.7 \pm 17.0$ & 0.299 \\
\hline $\begin{array}{l}\text { Number of vessels } \\
\text { with thick walls* } \\
(<3,4-5,[?] 6,)\end{array}$ & $\begin{array}{l}<3: 7 / 23(30.4 \%) \\
4-5: 15 / 23(65.2 \%) \\
{[?] 6: 1 / 23(4.3 \%)}\end{array}$ & $\begin{array}{l}<3: 0 / 17(0 \%) 4-5: \\
\text { 16/17 (94.1\%) [?]6: } \\
1 / 17(5.9 \%)\end{array}$ & $\begin{array}{l}<3: 1 / 15(6.7 \%) \\
4-5: 12 / 15(80 \%) \\
{[?] 6: 2 / 15(13.3 \%)}\end{array}$ & 0.039 \\
\hline $\begin{array}{l}\text { Wall of the } \\
\text { thickest vessel, } \\
\mu \mathrm{m} \text { (range) }\end{array}$ & $60(22-862)$ & $51(21.4-128)$ & $53(24.3-149.2)$ & 0.913 \\
\hline $\begin{array}{l}\text { Exterior fascia } \\
\text { thickness, }{ }^{* *} \mu \mathrm{m} \\
\text { (range) }\end{array}$ & $251(80-716)$ & $196(67.6-652)$ & $384.5(151-910.4)$ & 0.006 \\
\hline $\begin{array}{l}\text { Presence of } \\
\text { intercellular } \\
\text { collagen, n }(\%)\end{array}$ & $2(8.7 \%)$ & $2(11.8 \%)$ & $12(80 \%)$ & 0.000 \\
\hline $\begin{array}{l}\text { Presence of } \\
\text { spiculer fibrosis } \\
\text { and/or bands of } \\
\text { collagen, n }(\%)\end{array}$ & $5(22 \%)$ & $8(47 \%)$ & $13(87 \%)$ & 0.000 \\
\hline
\end{tabular}

*Thickness $>100 \mu \mathrm{m} .{ }^{* *}$ Measurements at the thickest point.

\section{FIGURE LEGENDS}

Figure 1. Cases showing increased fibrous tissue in the septae surrounding fat lobules (a), around the blood vessels (b) and in the Gerota fascia (c). Spicular infiltration of collagen into the fat lobules can be noted (a: Masson's trichrome stain x 200; b: Masson's trichrome stain x 100; c: H-E stain x 100).

Figure 2. Intercellular collagen deposition was noted in some cases expanding the distance between individual adipocytes (Left: H-E stain x 100; Right: Masson's trichrome stain x 400). 

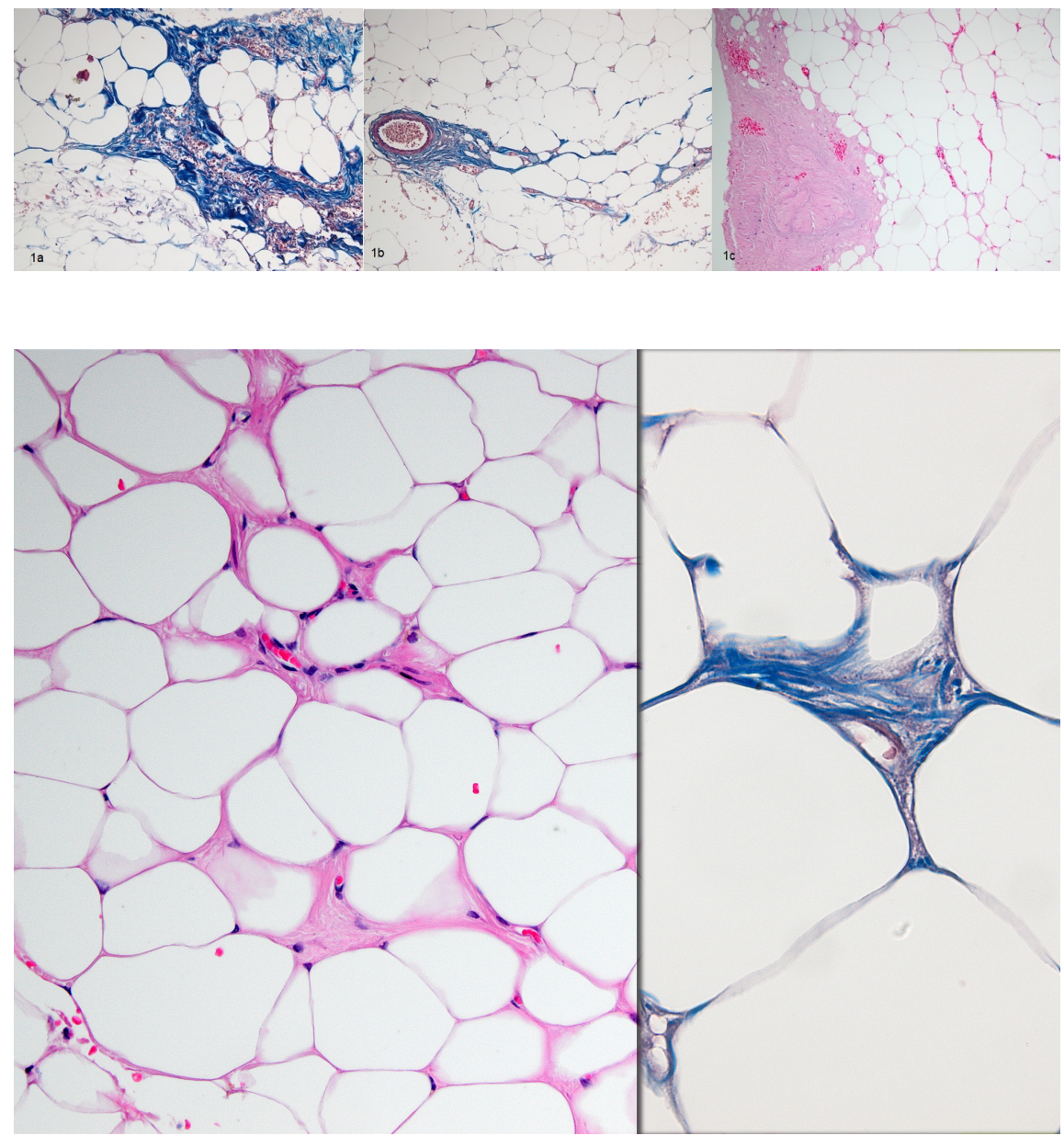

\section{Hosted file}

Table.pdf available at https://authorea.com/users/399372/articles/511928-pathologyassociated-with-adherent-perirenal-fat-and-its-clinical-effect 